\title{
INTEGRATION OF ACTIVE SENSORS FOR GEOMETRIC ANALYSIS OF THE CHAPEL OF THE HOLY SHROUD
}

\author{
Barba S. ${ }^{1, *}$, di Filippo A. ${ }^{1}$, Limongiello M. ${ }^{1}$, Messina B. ${ }^{1}$ \\ ${ }^{1}$ Department of Civil Engineering, University of Salerno, Italy - (sbarba, anddifilippo1, mlimongiello, bmessina)@unisa.it
}

\author{
Commission II, WG II/8
}

KEY WORDS: TLS, SLAM, Mobile Mapping Systems, Accuracy and Precision Assessment, Cultural Heritage.

\begin{abstract}
:
The digital acquisition of Cultural Heritage is a complex process, highly depending on the nature of the object as well as the purpose of its detection. Even if there are different survey techniques and sensors that allow the generation of realistic 3D models, defined by a good metric quality and a detail consistent with the geometric characteristics of the object, an interesting goal could be to develop a unified treatment of the methodologies. The Chapel of the Holy Shroud, with its intricate articulation, becomes the benchmark to test an integrated protocol between a terrestrial laser scanner (TLS) and a wearable mobile laser system (WMLS) based on a SLAM approach. In order to quantify the accuracy and precision of the latter solution, several forms of comparison are proposed. For the case study the ZEB-REVO, produced and marketed by GeoSLAM, is tested. Computations of cloud-to-cloud (C2C) absolute distances, comparisons of slices and extractions of planar features are performed, using stationary laser scanner (Faro Focus ${ }^{\mathrm{S}} \mathrm{S} 350$ ) as a reference. Finally, the obtained results are reported, allowing us to assert that the quality of the WMLS measurements is compatible with the data provided by the manufacturer, thus making the instrumentation suitable for certain specific applications.
\end{abstract}

\section{INTRODUCTION}

\subsection{Sensor integration for Cultural Heritage documentation}

The knowledge construction, as theorized by Aristotelian gnosis, finds its incipit in the sensorial perception of substance and materializes in an inductive process that, thanks to memory and experience, reaches the interpretation of non-substance, of the intelligible, producing a cultural enrichment. From this point of view, being witness to an ancient creative action transcends the pure substance aesthetics. A testimony acquired from the past and, therefore, the basis of the people identity, but also a present experience and the genesis of the community's future.

It is then desirable to consider that the Cultural Heritage enhancement does not end with the preservation of its materiality, but it is fully realized only on condition that the community can enjoy the asset and its knowledge. Principles already established by the numerous Charters for the Restoration that trace the real motivation of conservation and safeguarding of heritage in the social function carried out, thus establishing the right both to information, to be guaranteed through the prompt communication, and to enjoyment, to be assured in the most appropriate forms (Niglio, 2012).

In this articulated panorama, the complex function performed by the digital survey can be framed. It constitutes the cognitive moment that precedes the decisional one and defines the operative process for the Cultural Heritage conservation. The use of techniques and technologies for digital acquisition in the architectural field has now reached widespread diffusion, mainly due to the ability to detect artefacts with great precision and to the possibility of generating digital informative models useful for the analysis, simulation, interpretation and conservation phases (Barba et al., 2019).

If the definition of the digital survey role is unequivocal, it is not the same for the solutions through which this function is concretized, delineating a constantly evolving panorama (Blais, 2004). Today it is widely accepted that a careful selection and integration of different surveying techniques is preferable to individual methods. For this reason, words such as multisensor, multi-scale, multi-resolution are increasingly frequent and in demand in the field of Cultural Heritage (Guidi et al., 2008; Lerma et al., 2011). With regard to the metric survey and, more generally, the management of spatial information, the scientific community, starting from the semantic aspect, has extensively dealt with the concepts of integration of techniques and data fusion (Wald, 1999). The literature provides important examples, such as the fusion of TLS and WMLS models (Rodríguez-Gonzálvez et al., 2017). Other experiences investigate procedures to register frames on point clouds generated by TLS (Forkuo et al., 2004). The approach for the integration of multiple acquisition systems involves three main components: the representation of information, the description of uncertainty and the optimization of the method. From an operational point of view, this translates into the fact that there are some main purposes in the integrated application of two or more tools: the increasing of information on the object and the validation, or often the improvement, of the level of overall accuracy and precision. In the first case, the model is solved through a first spatial acquisition that is enriched with progressive additions that allow a better readability. The second and more complex aspect concerns the overall quality of the model, a very delicate issue for those involved in threedimensional surveying for documentation, restoration, replication and conservation purposes (Beraldin, 2004).

\subsection{Aims of the proposal and case study}

The present work proposes the integration of two different sensor systems. On the one hand, a TLS, whose application in the architectural digitization is more than consolidated and whose procedural pipeline is perfectly defined in the literature. On the other hand, a WMLS based on the SLAM approach (Stachniss et al., 2016), whose application in this sector is certainly more recent and lends itself to experimentation and reflection. The beginning of the research is represented by the observation that there is no relevant technique that allows optimal results in terms 
of accuracy, precision and photorealism in all work conditions. For this reason, the need is felt to orientate towards the integration of different techniques, in order to maximize results and reduce efforts. The benchmark of the experimentation is the Chapel of the Holy Shroud, one of the most important symbols of the Italian Baroque. This unique and magnificent place was strongly desired by Carlo Emanuele I of Savoy and the work started in 1607 . Three were the main construction sites that followed and the last one was emblematic, led by the architect and Teatino frair Guarino Guarini, a disciple of the architect Francesco Borromini. In the night between 11 and 12 April 1997, a fire affected the structure. It deeply damaged the building, requiring a long and demanding architectural and structural restoration. This intervention, one of the most complex within this discipline, recently finished giving back to humanity this splendid masterpiece.

Externally, the Chapel looks like a building with a square plan that interpenetrates both the Cathedral and the Royal Palace. Above the base rises a polygonal brick tambour with six large arched windows (Figure 1), framed by pilasters and protected by a roof that softly settles on the arches. Above it, there is a pseudodome with six rows of bows, supported by ribs on which numerous stone urns are installed (Messina, 2010). Among the ribs, arcuate lines draw numerous openings up to the terminal part of the dome, which rests on a small circular tambour (extraneous to the original design, which provided a helical cusp). If the articulation of the upper levels represents the highest expression of Guarini's Baroque genius, the same care has been reserved for the composition of the interior space: on the sides of the main altar of the Cathedral there are two portals in black marble that introduce two dark staircases with low semi-circular steps. At the end of the two staircases, there are two parallel circular vestibules bordered by black marble columns. They lead to the Chapel, with a circular plan. In the centre stands the Baroque altar that kept, in a silver and glass case, the Holy Shroud (Fittipaldi, 2014).

Such a complex structure requires the integration of several sensors, different for both acquisition procedure and data processing. Paths acquired with the SLAM approach join the TLS stations (with an average resolution of $6 \mathrm{~mm}$ to 10 meters), allowing the digitization of the tunnels that, crossing the masonry, connect the different levels of the Chapel. The

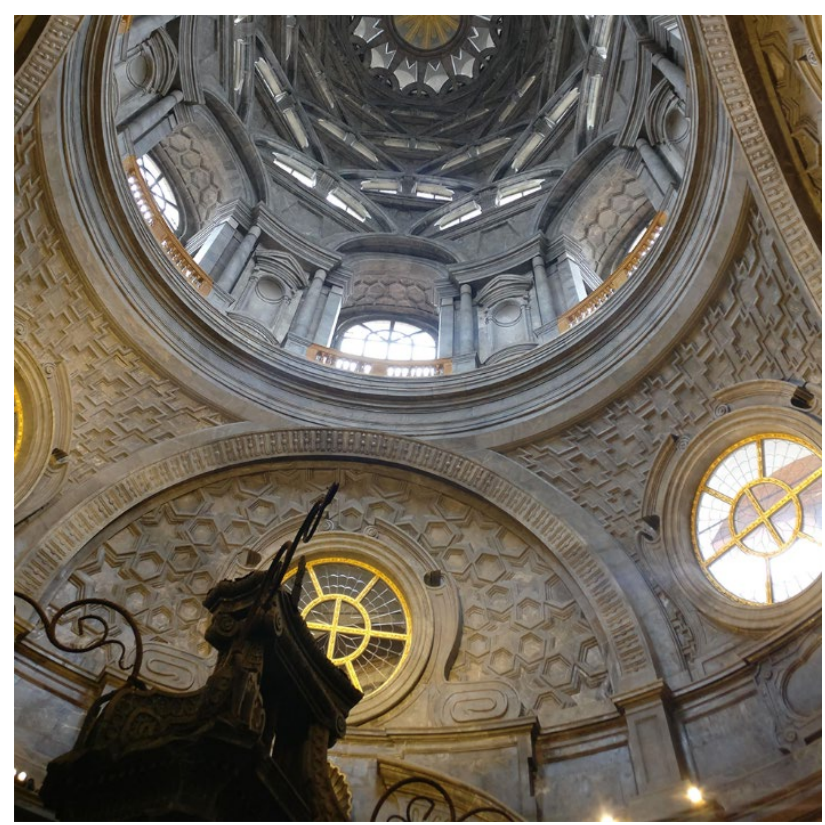

Figure 1. Pseudo-dome and tambour bottom view flexibility of the SLAM approach is added to the high accuracy and precision of the TLS systems, making it possible to enrich the final digital model with details undetectable by stationary tools due to an excessive intricacy of the paths. Obviously, as already stated, the fusion of models characterized by different resolution requires deep testing to verify compatibility. The subsequent sections of the manuscript are specifically aimed at proving the adopted procedure. Section 2 briefly describes the characteristics of the employed instrumentation. Section 3 presents the methods of data acquisition and management, defining a procedural pipeline. Section 4 reports the results obtained from the comparison phase of the homologous models and finally Section 5 closes this manuscript with the necessary reflections and conclusions.

\section{MATERIALS}

\subsection{Faro Focus ${ }^{\mathrm{s}}$ S350}

The S350 is a stationary laser scanner of the Continuous Wave - Frequency Modulation (CW-FM) type. The system is able to measure with great precision the direction of pointing, in addition to a distance meter that emits continuous light radiation. This one, thanks to a coding of the frequencymodulated light signal that allows the identification of a phase shift between the emitted wave and the recorded one, guarantees the indirect calculation of the time of flight and therefore of the distance. This solution guarantees a stated relative accuracy of $1 \mathrm{~mm}$.

\subsection{GeoSLAM ZEB-REVO}

The ZEB-REVO consists of a 2D time-of-flight laser range scanner rigidly coupled to an inertial measurement unit (IMU) mounted on a motor drive. The motion of the scanning head on the motor drive provides the third dimension required to generate 3D information. A simultaneous localization and mapping (SLAM) algorithm combines the 2D laser scan data with the IMU data to generate accurate $3 \mathrm{D}$ point clouds, employing a full SLAM approach (Grisetti et al., 2010; Bosse et al., 2012). Regarding accuracy, the manufacturer declares a value of $1-3 \mathrm{~cm}$ in relative terms and $1-30 \mathrm{~cm}$ in absolute positioning for a 10 -min scan, with a single loop closing.

\section{METHODS}

\subsection{Survey design and acquisition}

3.1.1 TLS: a survey design should be first define the positions of TLS stations, so that the whole object coverage at requested spatial resolution could be guaranteed. Considering that the Chapel has a maximum internal diameter of about $19 \mathrm{~m}$ at the level of the altar and assuming to place a station in correspondence of the centre, the instrument has been set so as to have a resolution of 6 $\mathrm{mm}$ to $10 \mathrm{~m}$. As far as the quality of the acquisition is concerned, for each point of the cloud three measurements are made in order to define the distance from the station as the average of the above measurements. Great attention has also paid to the problem of environmental occlusions, due to the presence of objects and architectural elements such as columns, friezes and decorations. In the first instance, 6 scans are acquired at the level of the altar and 6 at the level of the tambour. The presence of the altar protection system, made of steel and glass, has required additional stations to ensure the completeness of the model of the altar: 3 of them are placed in the intermediate choirs and 4 inside the barrier (Figure 2 - plans from "Musei Reali Torino"). With the features listed above, the TLS acquisition campaign has taken almost 5 hours, with a single-scan time of approximately 12 minutes. 

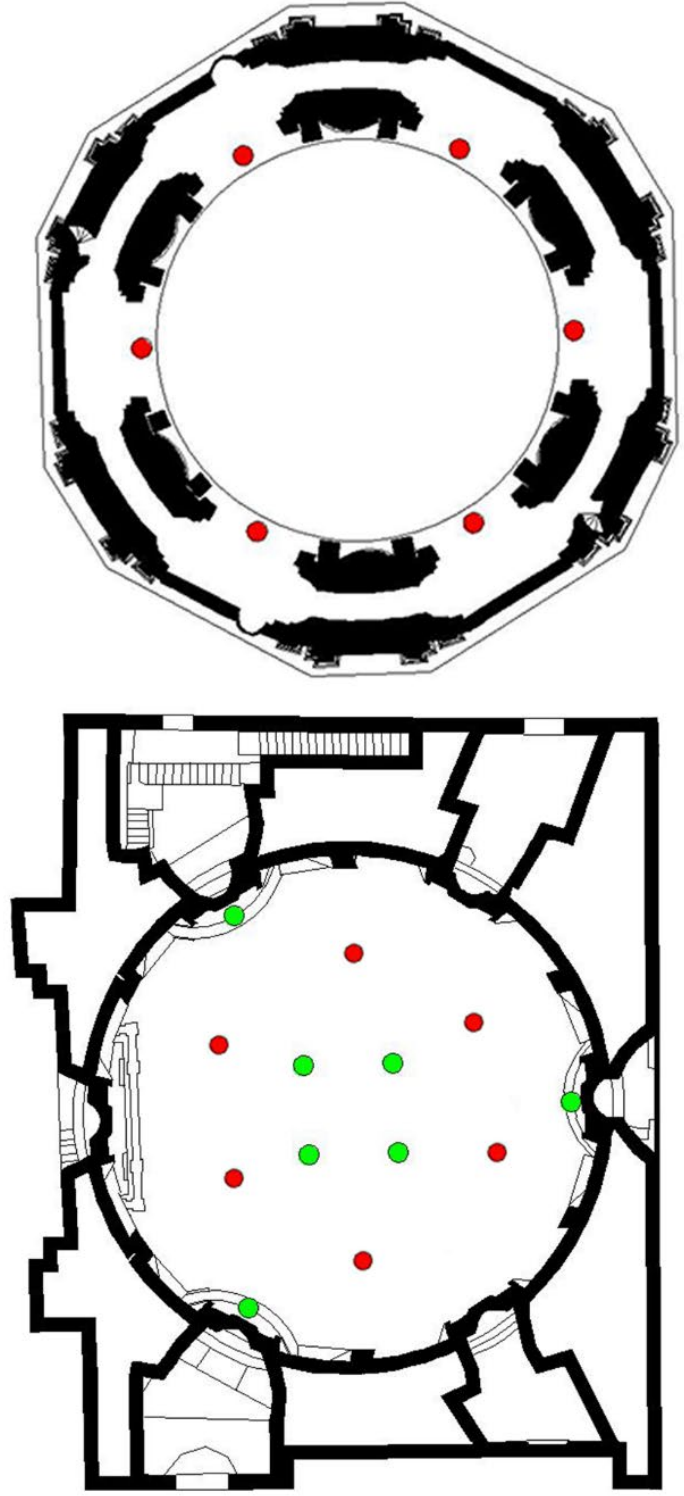

- First group of TLS stations

Second group of TLS stations

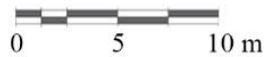

Figure 2. TLS stations at the altar (down) and tambour (up) level

3.1.2 WMLS: systems that use SLAM algorithms to generate final models require special attention when planning acquisitions. More than any other solution, in fact, the quality of the produced data is highly dependent on how the acquisition campaign is conducted (Di Filippo et al., 2018). It is important to inspect the site of interest in order to identify critical areas not detected during planning and remove any obstacles along the way. In addition to the focus on poorly referenced environments, transition areas and forward speeds, it should be remembered that full SLAM systems, such as the one employed, require the self-intersection of paths to ensure an appropriate redistribution of the accumulated errors. At least one loop must be closed, although it is advisable to plan routes with several self-intersections. With these concepts in mind, the acquisition campaign is organised in this way (Figure 3):

- first path, including the lower level of the altar and the sculptural groups;

- second path, with a part of the lower level and the corridors of the Doge's Palace;
- third path, containing the corridors of the building and a good part of the tunnels connecting to the upper level;

- fourth path, including the tunnels and the tambour walkway.

Digitizing the entire scene has taken 1 hour, about $1 / 5$ of the time needed to complete the TLS campaign, but covering a larger area.

\subsection{Post-processing: point cloud registration}

The processing of the raw data, coming from the acquisition phase, starts from the registration of the point clouds. Regardless of the employed survey technique, the solutions for registration proposed below are all based on the ICP algorithm (Besl et al., 1992; Chen et al., 1992). The proposed version is the standard one and solves an $L_{2}$-error minimization problem, defined as follows. Let two 3D point sets $X=\left\{\mathbf{x}_{i}\right\}, i=1, \ldots, M$ and $Y=\left\{\mathbf{y}_{i}\right\}, j=1, \ldots, N$, where $\mathbf{x}_{i}, \mathbf{y}_{j} \in \mathbb{R}^{3}$ are point coordinates of the aligned point set and reference point set respectively. The aim is to estimate a rigid motion with rotation $\mathbf{R}$ and translation $\mathbf{t}$, which minimizes the following $L_{2}$ error $E$ :

$$
E(\mathbf{R}, \mathbf{t})=\sum_{i=1}^{M} e_{i}(\mathbf{R}, \mathbf{t})^{2}=\sum_{i=1}^{M}\left\|\mathbf{R} \mathbf{x}_{i}+\mathbf{t}-\mathbf{y}_{j^{*}}\right\|^{2}
$$

where $e_{i}(\mathbf{R}, \mathbf{t})$ is the per-point residual error for $\mathbf{x}_{i}$. The point $\mathbf{y}_{j *} \in Y$ is denoted as the optimal correspondence of $\mathbf{x}_{i}$, which in the context of ICP is the closest point to the transformed $\mathbf{x}_{i}$ in $Y$ :

$$
j^{*}=\underset{j \in\{1, \ldots, N\}}{\operatorname{argmin}}\left\|\mathbf{R} \mathbf{x}_{i}+\mathbf{t}-\mathbf{y}_{j}\right\|
$$

Given initial transformation $\mathrm{R}$ and $\mathrm{t}$, the ICP algorithm iteratively solves the above minimization via alternating between estimating the transformation in Equation (1), and finding the closest-point matches by Equation (2). Due to such iterative nature, ICP can only guarantee the convergence to a local minimum. For this reason, all the registration procedures are visually proven. The

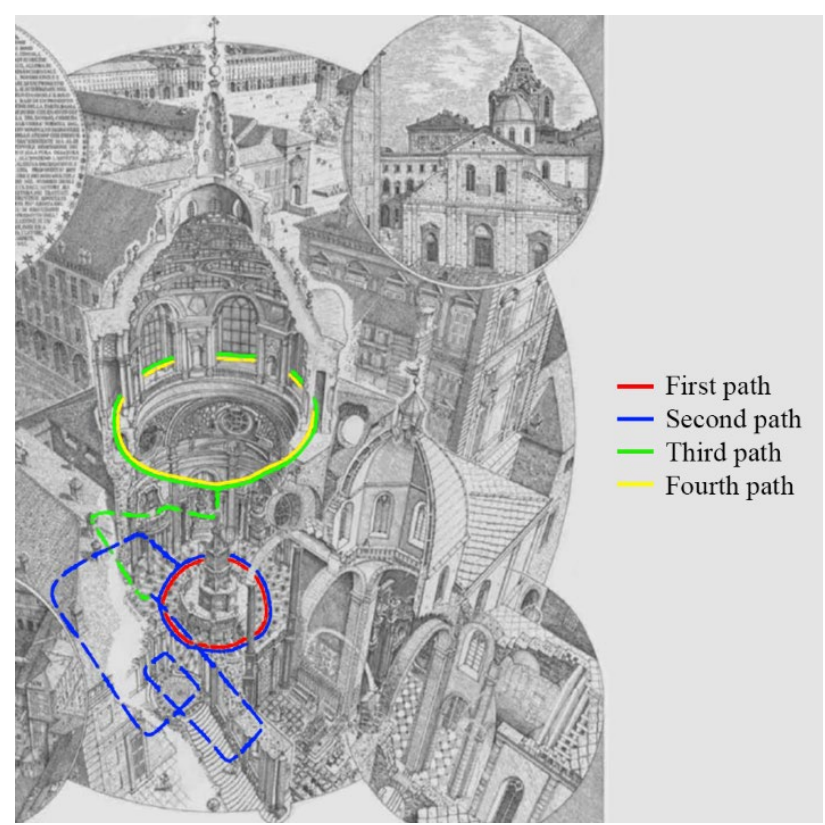

Figure 3. WMLS acquisition paths (on drawing by Capra A.) 
registration error is expressed through the RMSE, or the RMS of the Euclidean distance between the matching point pairs used at the last step of the alignment process in the two scans. The RMSE can be expressed as follows:

$$
\operatorname{RMSE}=\sqrt{\frac{E(\mathbf{R}, \mathbf{t})}{M}}
$$

For each ICP application a maximum number of matches was set equal to 5 million and a number of iterations for the process equal to 100 .

3.2.1 TLS scan registration: these data are used to create a reference network (our ground truth), indispensable for performing a quality assessment on the WMLS. The clouds are characterized by a high degree of overlap and for this reason are registered employing a global bundle adjustment procedure, accomplished after a top view based pre-registration. Given the set of scans, the algorithm searches for all the possible connections between the pairs of point clouds with overlap (Figure 4). For each connection, a pairwise ICP is performed and the best matching point pairs between the two scans are saved. A final non-linear minimization is run only among these matching point pairs of all the connections. The global registration error of these point pairs is minimized, having as unknown variables the scan poses (Santamaría et al., 2011). The maximum value of the RMSE on the individual registration pairs is about $1.12 \mathrm{~cm}$. Even the original versions of the scans are preserved to conduct more checks.

3.2.2 WMLS data registration: due to the low overlap between the four acquisition paths, a progressive registration approach based on an ICP pairwise algorithm is employed, each time choosing the reference scan (Figure 5). All preceded by a manual raw alignment. The maximum value of the RMSE on all the registration pairs, defined above, is about $1.74 \mathrm{~cm}$. The idea of defining a pipeline for the registration of WMLS data that does not resort to TLS scans arises from the observation that it is not always possible to have homologous models deriving from different systems to perform accuracy and precision checks. For this reason, the workflows for the two systems are kept separate until comparison. The individual paths are maintained both disjoined for a $\mathrm{C} 2 \mathrm{C}$ comparison with the final TLS model, both merged for the section extraction and the application of algorithm for adapting graphic primitives.

\subsection{Accuracy and precision assessment protocol}

The comparison between homologous models, produced with the TLS technique and the SLAM approach, is performed according to different modalities. Before proceeding, it may be useful to identify the error components when comparing point clouds:

- the component of position of the cloud, depending on the technology used in the acquisition phase;

- the component of registration among the point clouds, depending on the technique used to define a common reference system;

- the errors depending on occlusions and on the process of discretization of the survey.

Once the error components have been defined, some algorithms are selected to perform the comparison. The main ones are presented below.

3.3.1 Direct C2C comparison with closest point technique: this method is the simplest and fastest direct 3D comparison method of point clouds, as it does not require gridding or meshing of the data, nor calculation of surface normal vectors. For each point of the analysed cloud, a closest point can be defined in the reference. In its simplest version, the surface change is estimated as the distance between the two points (Lague et al., 2013). An improvement may consist of local modelling of the reference mesh. This technique is also used in cloud matching techniques such as the ICP. The difference lies in the fact that the computation of the closest point for the ICP is performed only on sample used to construct the matching pairs. In the case of a comparison, instead, all the points of the analysed cloud are considered. This type of distance is sensitive to the cloud roughness, outliers and point spacing. For this reason, the technique is developed for rapid change detection on very dense point clouds (like out TLS model) rather than accurate distance measurement.

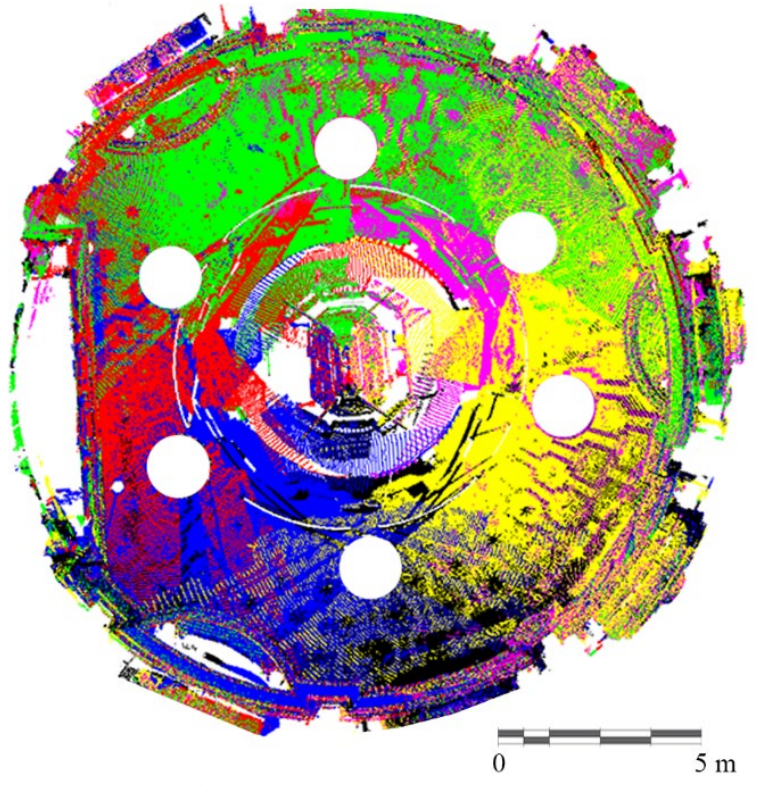

Figure 4. TLS global registration at the altar level

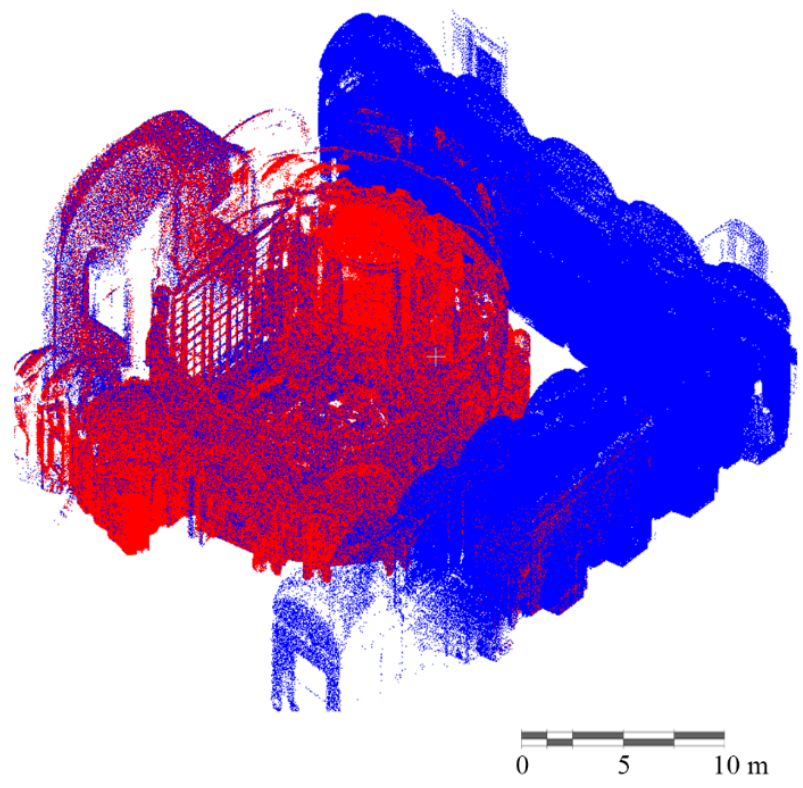

Figure 5. First (red) and second (blue) WMLS path registration 
3.3.2 RANSAC for point cloud shape detection: the RANSAC paradigm extracts shapes by randomly drawing minimal sets from the point data and constructing corresponding shape primitives (Schnabel et al., 2007). A minimal set is the smallest number of points required to uniquely define a given type of geometric primitive. The resulting candidate shapes are tested against all points in the data to determine how many of the points are well approximated by the primitive. After a given number of trials, the shape, which approximates the most points, is extracted and the algorithm continues on the remaining data.

3.3.3 Cloud-to-mesh (C2M) distance: this approach is the most common. Surface change is calculated by the distance between a point cloud and a reference 3D mesh or theoretical model (Cignoni et al., 1998). This approach works well on flat surfaces as a mesh corresponding to the average reference point cloud position can be constructed. However, creating a surface mesh is complex for point clouds with significant roughness at all scales or missing data due to occlusion. It generally requires time-consuming manual inspection. Interpolation over missing data introduces uncertainties that are difficult to quantify. Mesh construction also smooths out some details that may be important to assess local roughness properties.

\section{EXPERIMENTAL RESULTS}

4.1.1 C2C absolute distance computation: this first analysis involves the extraction of the region occupied by the altar from the previously registered homologous models, with subsequent C2C comparison. In particular, the first three WMLS paths are employed (the only ones containing the altar), each of them compared with the merged TLS cloud (used as reference). For the study, no local cloud modelling is employed due to the high surface density of the TLS cloud, but simply the C2C absolute distance is calculated (Figure 6). In all cases, a mean distance value of $1.30 \mathrm{~cm}$ is obtained (corresponding to the mean absolute error - MAE - value) and a standard deviation of $1.20 \mathrm{~cm}$. The value of the MAE, which defines the accuracy in terms of magnitude, is within the limits of relative accuracy declared by the manufacturer, equal to $3 \mathrm{~cm}$. The same procedure is used to compare the paths with a single TLS scan, after a pairwise ICP registration. This is to verify if a hypothetical residual error in the TLS data registration process is able to influence the comparison. The results are reported in Table 1.

\begin{tabular}{|c|c|c|}
\hline Path & \multicolumn{2}{|c|}{ C2C absolute distance (cm) } \\
\hline & Mean & Std. dev. \\
First & 1.66 & 1.72 \\
Second & 1.86 & 2.03 \\
Third & 1.82 & 2.13 \\
\hline
\end{tabular}

Table 1. Distance between the WMLS paths and a single TLS

$$
\text { scan }
$$

A slight increase in values can be observed. This is attributable to the presence, in the TLS scan, of some voids produced by environmental occlusions in the acquisition phase. As a result, there is no perfect overlap between homologous models, with higher distance between clouds in small located areas. The values obtained are however compatible with the relative accuracy of the instrument

4.1.2 Slice comparison: A second analysis involves the export of slices from the registered homologous models. For the case study, four pairs of slices are examined, two at the altar level and two at the tambour level with a pitch of $5 \mathrm{~m}$ and a thickness of $5 \mathrm{~cm}$, subsequently compared with a point-to-point distance algorithm. The WMLS paths are merged and, before the comparison, two different types of registration with the TLS data are performed: a registration with the full TLS model, for an error compensation on the acquired scene (Figure 7) and a registration with the TLS scans at the altar level only, in order to highlight any error accumulation along the paths. Table 2 shows the results.

\begin{tabular}{|c|c|c|c|c|}
\hline \multirow{2}{*}{ Slice } & \multicolumn{2}{|c|}{$\begin{array}{c}\text { C2C absolute } \\
\text { distance (cm) - full } \\
\text { TLS registration }\end{array}$} & \multicolumn{2}{c|}{$\begin{array}{c}\text { C2C absolute } \\
\text { distance (cm) - altar } \\
\text { level registration }\end{array}$} \\
\hline & Mean & Std. dev. & Mean & Std. dev. \\
Altar $(0.5 \mathrm{~m})$ & 1.74 & 1.90 & 1.52 & 1.24 \\
Altar $(5.5 \mathrm{~m})$ & 1.56 & 1.44 & 1.48 & 1.04 \\
Tambour $(25.5 \mathrm{~m})$ & 1.39 & 1.54 & 2.23 & 1.98 \\
Tambour $(30.5 \mathrm{~m})$ & 1.59 & 1.68 & 2.66 & 2.02 \\
\hline
\end{tabular}

Table 2. Slice comparison after two different types of registration

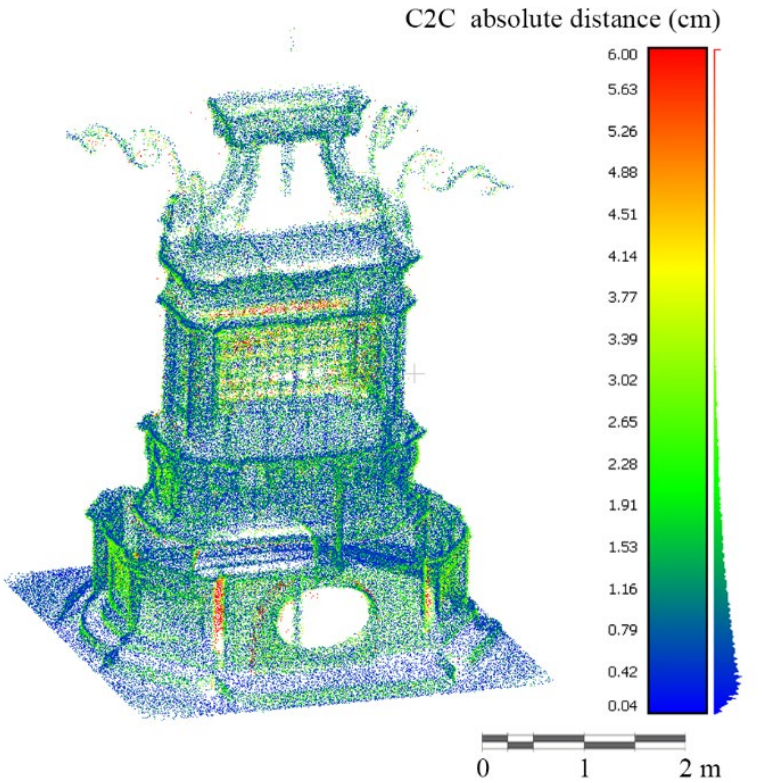

Figure 6. $\mathrm{C} 2 \mathrm{C}$ distance between the second path and merged TLS

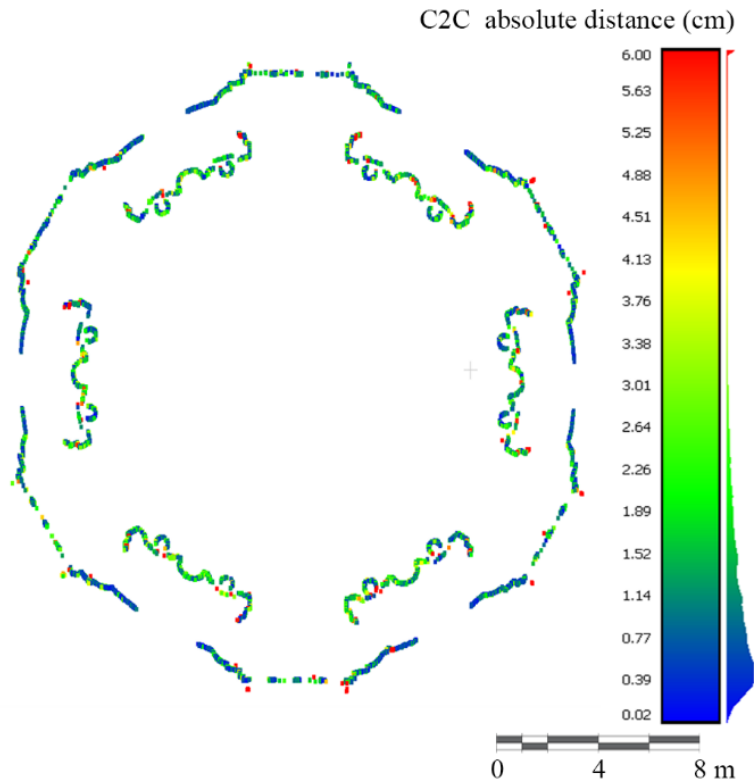

Figure 7. C2C slice $(25.5 \mathrm{~m})$ comparison after full TLS registration 
The comparison of the data in the table shows how, going from a global registration to one that only affects the level of the altar, the MAE at the lower level decreases as it increases for the slices placed at greater altitude. The values obtained are, however, compatible with the relative accuracy declared by the instrument manufacturer, equal to $3 \mathrm{~cm}$. This allows us to conclude that the possible accumulation of errors along the acquisition paths is negligible.

4.1.3 Planar feature extraction: the third analysis involves the extraction of flat elements from the two registered homologous models (merged WMLS and fused TLS cloud). By employing a RANSAC algorithm, planar graphic primitives are fitted to the reference TLS cloud and a C2M signed distance between merged WMLS paths and planes in computed. In order to simplify the analysis of the measurements, the normal plane vectors are oriented towards the canter of the Chapel.

A total of 10 reliable primitives are extracted, 4 at the altar level (A) and 6 at the tambour level (T) (Figure 8). Columns 2 and 3 of Table 3 report statistics on the calculated distance.

So that the analysis we just conducted makes sense, it is necessary to verify the hypothesis of flatness of the elements extracted from the clouds. For this reason, the $\mathrm{C} 2 \mathrm{M}$ signed distance between the TLS cloud and the best fit plane is calculated. Columns 4 and 5 of Table 3 show the results.

\begin{tabular}{|c|c|c|c|c|}
\hline \multirow[t]{2}{*}{ Plane } & \multicolumn{2}{|c|}{$\begin{array}{c}\text { C2M signed } \\
\text { distance }(\mathrm{cm}) \text { - } \\
\text { WMLS }\end{array}$} & \multicolumn{2}{|c|}{$\begin{array}{c}\text { C2M signed } \\
\text { distance }(\mathrm{cm})- \\
\text { TLS }\end{array}$} \\
\hline & Mean & Std. dev. & Mean & Std. dev. \\
\hline $1(\mathrm{~A})$ & 0.07 & 1.31 & 0.00 & 0.41 \\
\hline $2(\mathrm{~A})$ & 0.10 & 1.37 & 0.00 & 0.24 \\
\hline 3 (A) & 0.10 & 1.42 & 0.00 & 0.23 \\
\hline $4(\mathrm{~A})$ & 0.04 & 1.49 & 0.00 & 0.57 \\
\hline $5(\mathrm{~T})$ & 0.12 & 1.21 & 0.00 & 0.52 \\
\hline $6(\mathrm{~T})$ & 0.03 & 1.27 & 0.00 & 0.40 \\
\hline $7(\mathrm{~T})$ & -0.01 & 1.09 & 0.00 & 0.33 \\
\hline $8(\mathrm{~T})$ & -0.03 & 1.19 & 0.00 & 0.36 \\
\hline $9(\mathrm{~T})$ & 0.00 & 1.34 & 0.00 & 0.48 \\
\hline $10(\mathrm{~T})$ & -0.03 & 1.30 & 0.00 & 0.53 \\
\hline
\end{tabular}

Table 3. Signed distance between clouds and reference planes

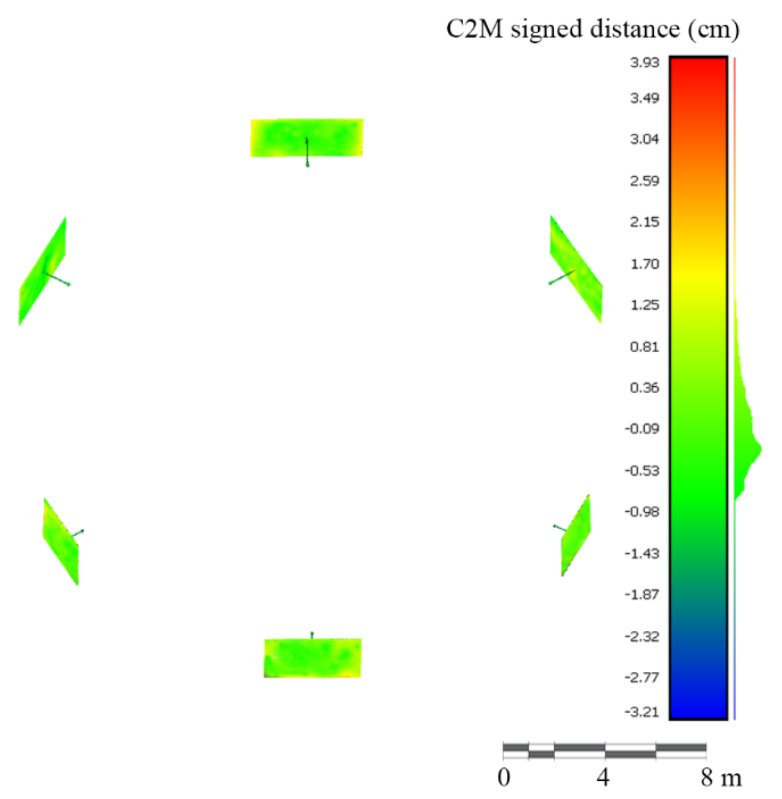

Figure 8. Tambour level features and histogram of TLS plane 6 (T)
An examination of the histograms related to the statistics shown in Table 3 for the WMLS allows us to state roughly that the distances with sign have a normal distribution (Figure 9). The average values of the distances (corresponding to the mean bias errors - MBE) are close to zero. This means that the bias is negligible. The standard deviations of the WMLS cloud are reasonably higher than those obtained from the TLS one. In general, the values are very similar to those of other studies in the literature (Maboudi et al., 2017; Sirmacek et al., 2016).

Another check is performed using the graphic primitives. By applying a plan of best fit also to the WMLS cloud, it has been calculated the angle formed by the normal unitary vector of the two homologous planes (TLS and WMLS). In every comparison the 2 tenths of a degree are never exceeded. Table 4 shows the results in detail.

\begin{tabular}{|c|c|c|c|}
\hline Plane & Angle $\left(^{\circ}\right)$ & Plane & Angle $\left(^{\circ}\right)$ \\
\hline $1(\mathrm{~A})$ & 0.15 & $6(\mathrm{~T})$ & 0.05 \\
$2(\mathrm{~A})$ & 0.11 & $7(\mathrm{~T})$ & 0.05 \\
$3(\mathrm{~A})$ & 0.18 & $8(\mathrm{~T})$ & 0.05 \\
$4(\mathrm{~A})$ & 0.13 & $9(\mathrm{~T})$ & 0.04 \\
$5(\mathrm{~T})$ & 0.02 & $10(\mathrm{~T})$ & 0.05 \\
\hline
\end{tabular}

Table 4. Angle between best fit homologous planes

\section{CONCLUSIONS}

In this paper, the quality of point clouds acquired by a wearable mobile mapping system, namely the handheld GeoSLAM ZEB-REVO, is tested in a controlled environment. Quantitative and qualitative analysis of the point clouds are performed using the point cloud of a Faro Focus ${ }^{\mathrm{S}} \mathrm{S} 350$ as reference. Overall, the measurement quality, reflected in the $\mathrm{C} 2 \mathrm{C}$ absolute distance to the reference model, the $\mathrm{C} 2 \mathrm{C}$ absolute distance between homologous slices and the signed distance from reference best fit planes is in line with expectations.

The computed MAE is always less than $2.00 \mathrm{~cm}$. This value, which defines the accuracy in terms of magnitude, is within the limits of relative accuracy declared by the manufacturer, equal to $3 \mathrm{~cm}$. Numerical calculations are always accompanied by a visual inspection to prove the results (Figure 10).

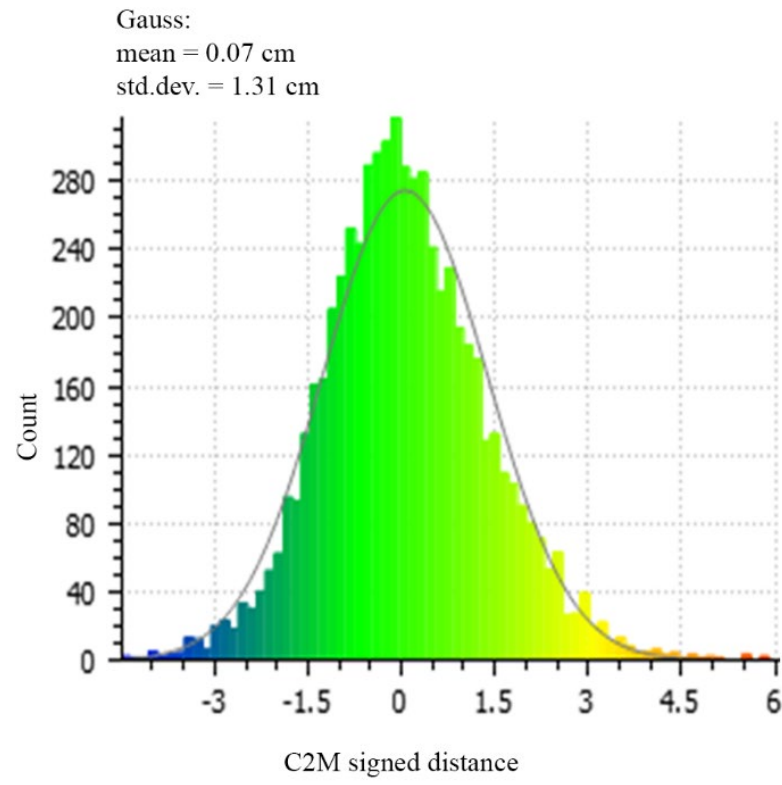

Figure 9. Signed distance histogram of WMLS plane 1 (A) 


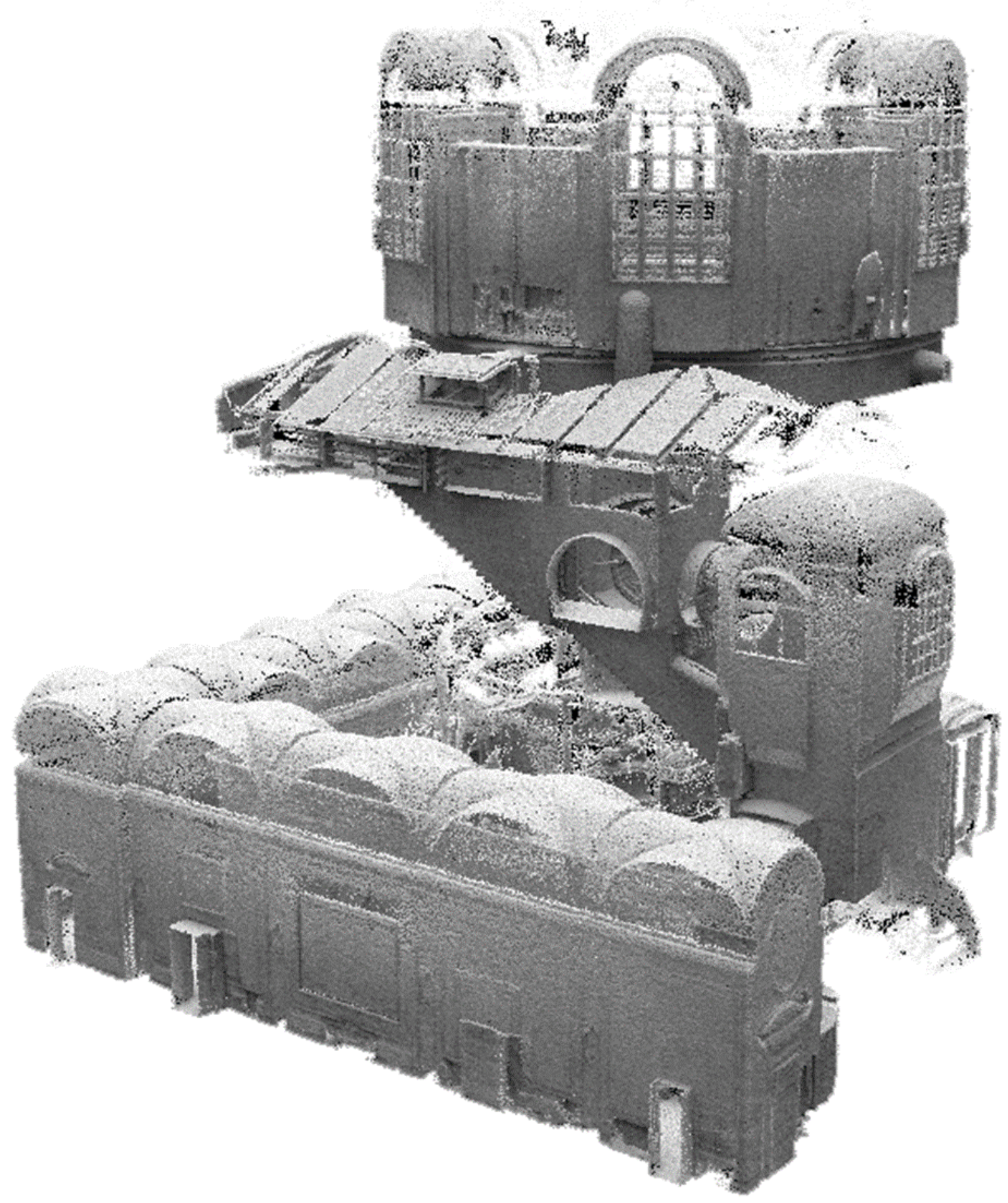

Figure 10. Perspective view of the tunnel connecting the first and the second levels of the Chapel (SLAM model)

With regard to the fit of plans on reference models, the standard deviation values for the WMLS signed distance, representative of an RMSE, are less than $1.50 \mathrm{~cm}$ and compatible with the results of similar studies (Maboudi et al., 2017; Sirmacek et al., 2016). The values of the MBE, close to zero, also show negligible bias. Furthermore, if a geometric entity like a plane is extracted from a point cloud for distance measurement and comparison, it is necessary to verify the planarity hypothesis. Finally, the angle between the normal unitary vectors of the homologous best fit plans is always less than 2 tenths of a degree. Future work will focus on improving statistical error analysis and building a structured database from the fusion of different sensor data.

\section{AKNOWLEDGEMENT}

The authors are indebted to Gennaro Miccio, secretary of the Ministry of Cultural Heritage and Activities for the Piemonte Region. His support has guaranteed the authors the possibility to operate the survey benefiting from the necessary support. A further thanks goes to Gianluca Ronga, for the enthusiasm shown during the collaboration with the authors.

Finally, a special thanks to Metrologia S.p.A. (www.metrologiaspa.com) and CAM2 (www.faro.com/it-it/) who provided respectively the ZEB-REVO and the Faro Focus ${ }^{\mathrm{S}}$ S350. 


\section{REFERENCES}

Barba, S., Barbarella, M., Di Benedetto, A., Fiani, M., and Limongiello, M. 2019. Comparison of Uavs Performance for a Roman Amphitheatre Survey: the Case of Avella (Italy). In: The International Archives of the Photogrammetry, Remote Sensing and Spatial Information Sciences, Vol. XLII-2/W11, pp. 179-186.

Beraldin, J. A. 2004. Integration of laser scanning and closerange photogrammetry - The last decade and beyond. In: Proceedings of the XXth ISPRS Congress, pp. 12-23.

Besl, P. J., and McKay, N. D. 1992. Method for registration of 3D shapes. In: Sensor fusion IV: control paradigms and data structures, Vol. 1611, pp. 586-606. International Society for Optics and Photonics.

Blais, F. 2004. Review of 20 years of range sensor development. Journal of electronic imaging, 13(1), pp. 231-44.

Bosse, M., Zlot, R., and Flick, P. 2012. Zebedee: Design of a Spring-Mounted 3-d Range Sensor with Application to Mobile Mapping. IEEE Transactions on Robotics, 28(5), pp. 1104-19. doi: 10.1109/TRO.2012.2200990.

Chen, Y., and Medioni, G. 1992. Object modelling by registration of multiple range images. Image and Vision Computing, 10(3), pp. 145-155. doi: https://doi.org/10.1016/0262-8856(92)90066-C.

Cignoni, P., Rocchini, C., and Scopigno, R. 1998. Metro: Measuring Error on Simplified Surfaces. In: Computer Graphics Forum. Vol. 17, No 2, pp. 167-174 doi: 10.1111/14678659.00236 .

di Filippo, A., Sánchez-Aparicio, L. J., Barba, S., Martín-Jiménez, J. A., Mora, R., and González Aguilera, D. 2018. Use of a Wearable Mobile Laser System in Seamless Indoor 3D Mapping of a Complex Historical Site. Remote Sensing, 10(12), 1897.

Fittipaldi, G. 2014. Spazio, forma e struttura nelle architetture di Guarino Guarini. Gangemi Editore Spa.

Forkuo, E. K., and King, B. 2004. Automatic fusion of photogrammetric imagery and laser scanner point clouds. In: The International Archives of Photogrammetry and Remote Sensing, Vol. 35, pp. 921-926.

Grisetti, G., Kummerle, R., Stachniss, C., and Burgard, W. 2010. A Tutorial on Graph-Based SLAM. IEEE Intelligent Transportation Systems Magazine, 2(4), pp. 31-43. doi: 10.1109/MITS.2010.939925.

Guidi, G., Remondino, F., Russo, M., Menna, F., and Rizzi, A. 2008. 3D Modeling of Large and Complex Site Using Multisensor Integration and Multi-resolution Data. In: Proceedings of the History and territory valorization event.

Lague, D., Brodu, N., and Leroux, J. 2013. Accurate 3D comparison of complex topography with terrestrial laser scanner: Application to the Rangitikei canyon (N-Z). ISPRS Journal of Photogrammetry and Remote Sensing, 82, pp. 1026. doi: https://doi.org/10.1016/j.isprsjprs.2013.04.009.

Lerma, J. L., Navarro, S., Cabrelles, M., Seguí, A. E., Haddad, N., and Akasheh, T. 2011. Integration of laser scanning and imagery for photorealistic 3D architectural documentation. Laser scanning, theory and applications, pp. 413-430.
Maboudi, M., Bánhidi, D., and Gerke, M. 2017. Evaluation of indoor mobile mapping systems. In: Proceedings of the GFaI Workshop 3D North East.

Messina, B. 2010. Geometria e simbolo: la cappella della Sacra Sindone. In: Disegnare il tempo e l'armonia. Il disegno di architettura osservatorio dell'universo. Alinea editrice, Firenze, pp. 692-697

Niglio, O. 2012. Le Carte del Restauro. Documenti e Norme per la Conservazione dei Beni Architettonici ed Ambientali. Aracne Editrice, Roma.

Rodríguez-Gonzálvez, P., Jiménez Fernández-Palacios, B., Muñoz-Nieto, L. Á., Arias-Sanchez, P., and Gonzalez-Aguilera, D. 2017. Mobile LiDAR System: New Possibilities for the Documentation and Dissemination of Large Cultural Heritage Sites. Remote Sensing, 9(3), 189. doi: 10.3390/rs9030189.

Santamaría, J., Cordón, O., and Damas, S. 2011. A comparative study of state-of-the-art evolutionary image registration methods for 3D modeling. Computer Vision and Image Understanding, 115(9), pp. 1340-1354. doi: https://doi.org/10.1016/j.cviu.2011.05.006.

Schnabel, R., Wahl, R., and Klein, R. 2007. Efficient RANSAC for Point-Cloud Shape Detection. In: Computer Graphics Forum, Vol. 26, No 2, pp. 214-226. doi: 10.1111/j.1467-8659.2007.01016.x.

Sirmacek, B., Shen, Y., Lindenbergh, R., Zlatanova, S., and Diakite, A. 2016. Comparison of Zeb1 and Leica C10 indoor laser scanning point clouds. ISPRS Ann. Photogramm. Remote Sens. Spat. Inf. Sci, 3, pp. 143-149.

Stachniss, C., Leonard, J. J., and Thrun, S. 2016. Simultaneous Localization and Mapping. In: Springer Handbook of Robotics. Springer International Publishing, pp. 1153-1176.

Wald, L. 1999. Some terms of reference in data fusion. IEEE Transactions on Geoscience and Remote Sensing, 37(3), pp. 1190-1193. doi: 10.1109/36.763269. 